\title{
Packing dynamics of powders at high temperature
}

\author{
Aurélien Neveu ${ }^{1, *}$, Filip Francqui ${ }^{1}$, and Geoffroy Lumay $^{2}$ \\ ${ }^{1}$ GranuTools, Awans, Belgium \\ ${ }^{2}$ GRASP Laboratory, CESAM research unit, University of Liège, Belgium
}

\begin{abstract}
In industrial powder processing, precise control of environmental temperature is difficult especially for large production facilities. A better knowledge of powder behavior modification due to temperature variation will help to improve product quality and consistency. From a fundamental point of view, the effect of temperature on powder flow and packing dynamics is still poorly understood. In particular, because temperature modifies the complex interplay between the different forces acting at the contact between the grains. The packing dynamics of a set of different powders (lactose, cacao and dried milk powder) has been investigated with a tapping experiment. After the filling procedure, the sample is heated and the evolution of the density is measured after each tap. We show that the packing dynamics is drastically influenced even for low temperature changes. Slight increase of temperature affects both the packing kinematics and the packing range characterised by the classical Hausner ratio analysis. Finally, the different physical mechanisms that could explain these modifications are discussed. The results of this preliminary study demonstrate the importance of temperature in powder behaviour and strongly motivate further investigations.
\end{abstract}

\section{Introduction}

Numerous industrial applications involve handling and processing of granular materials and fine powders. However, providing a complete description of their behavior is still challenging [1-4]. Due to the lack of complete theoretical models, the understanding of powder behavior has to rely on characterization of the properties of the grains (size and shape distribution, roughness, porosity, ...) and to the behavior of the assembly of grains (packing dynamics, flow, agglomeration, segregation, ...) [5-7]. Moreover, the environmental conditions (temperature and relative humidity) are also key parameters that influence powder behavior and have to match those of the process to allow reliable predictions.

Many parameters are influencing the behavior of powders such as steric repulsion, friction forces, cohesive forces and interaction with the surrounding gas. Both the surface state (rough or smooth surface) and the chemical nature of the grains influence the friction forces. Cohesive interactions may be due to the presence of liquid bridges [8-11], electrostatic charges [12], van der Waals interactions $[17,18]$ or more rarely magnetic dipole-dipole interactions [19-22]. Environmental conditions can modify the predominance of these grain interactions. Indeed, moisture is known to influence both surface grains conductivity and capillary bridges formation leading to a modification of the static and dynamic behavior. Furthermore, empirical observation seams to indicate that the temperature is affecting the interaction between the grains as well as grains breakage behavior [23].

*e-mail: Aurelien.Neveu@ granutools.com

A video is available at https://doi.org/10.48448/3r8k-tx81
Precisely controlling the powder temperature during a process requires costly equipment and is almost impossible for large production facility. Seasonal changes and production in different sites over the globe induce unavoidable variations of the environmental conditions at which the powder is processed. Therefore, understanding the influence of temperature on the powder behavior is of great interest to predict decrease of process performance and elaborate optimal processing conditions. However, previous studies have focused on processes that involve high temperature and mainly considered influence of temperature on polymer [13-15] and metal powders [16].

Packing dynamics characterization gives useful information on the powder properties which can be related to its flowability. Based on fundamental research results [17, 21, 24-28], the classical tapped density measurement methods [29] has been improved with the development of the GranuPack [7, 30]. After each tap, the density is measured accurately with the use of an inductive sensor, given access to the whole compaction curve. In addition to the classical parameters like bulk and tapped density, Hausner ratio and Carr index, dynamical parameters are extracted from the packing curve to characterize powder flowability.

In the present study, we explore the influence of temperature on the packing dynamics of common food and lactose powders. A novel characterization method allowing to investigate the packing dynamics at different temperature is presented. We show that even low variation of temperature leads to significant changes in the powder packing dynamics. The importance of evaluating the effect of temperature even for processes that do not involve powder heating is thus highlighted. These new results are 
expected to motivate the development of future investigations to better understand the underlying physical mechanisms that lead to important changes in powder flowability due to temperature.

\section{Experimental set-up}

\subsection{Materials}

For this study, three very common and commercially available powders have been selected: a cocoa, a dried milk and a lactose. The lactose powder is a milled monohydrate lactose grade (Pharmatose ${ }^{\circledR} 200 \mathrm{M}$, DFE Pharma) used as an excipient for tablet production of pharmaceutical blends. The lactose used in this study has a D50 of $37.8 \mu \mathrm{m}(D 10=4.4 \mu \mathrm{m}, D 90=111.3 \mu \mathrm{m})$. No information are available on the particle sizes of the cocoa and dried milk powders. As this work is intended to highlight the influence of temperature on the macroscopic behavior of the powders, changes in the microscopic grain properties (size, shape, chemical composition) have not been investigated.

\subsection{Method}

The need to evaluate temperature influence on powder packing dynamics has motivated the adaptation of the GranuPack instrument to perform measurement at high temperature. The measurement cell is surrounded by an electrical heating jacket coupled with a thermocouple to measure the temperature of the cell. The heat generated by the cell does not allow the use of the inductive sensor present in the classical GranuPack to measure the pile height $h$, which has been replaced by a laser sensor situated farthest from the hot sample (see figure 1). The new measurement cell is able to heat up the sample till $200^{\circ} \mathrm{C}$. This set-up has already been applied successfully to the investigation of the influence of temperature on polymer powders [15]. The ambient temperature in the laboratory was $20^{\circ} \mathrm{C} \pm 2^{\circ} \mathrm{C}$ at a relative humidity of $41 \% \pm 6 \%$. Before the measurement the sample is weighted with a precision scale.

At the start of the measurement the powder is automatically poured in a metallic tube following an initialization process described in previous publications [7, 30]. To increase measurement accuracy a light hollow cylinder $(12 \mathrm{~g})$ is gently placed on top of the powder bed to provide a flat powder/air interface. The presence of the hollow cylinder may slightly influence the achieved bulk density. However, this effect is considered low and identical for the tested powders and thus will not be considered in this comparative study. This also helps to reduce thermal dissipation during the measurement. The heating of the cell then begin to increase the temperature to the selected temperature. After target temperature is reached a waiting period of 30 minutes is performed to guarantee a uniform temperature inside the sample [15].

The taps are applied by rising the tube containing the powder sample up to a height of $\Delta Z$ then let it experience free falls. After each tap, the laser sensor measure

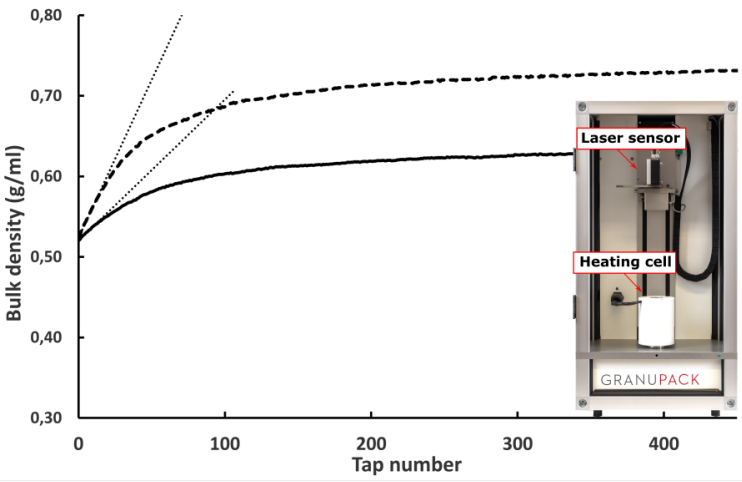

Figure 1. Packing dynamics curves obtained with the GranuPack instrument for the lactose powder at $20^{\circ} \mathrm{C}$ (dashed line) and $120^{\circ} \mathrm{C}$ (solid line). The slope of the initial stage of packing (dotted lines) highlight the slowdown of packing kinematics at higher temperature. Insert: picture of the GranuPack instrument.

the height $h$ of the powder bed to compute the volume $V$ of the pile. As the powder mass $m$ is known, the density $\rho$, the ratio between the mass $m$ and the powder bed volume $V$, is computed and plotted after each tap on a packing curve. The results presented here were obtained with $35 \mathrm{ml}$ powder sample volume, a free fall of $\Delta Z=1 \mathrm{~mm}$ and a sequence of 500 taps.

Powder packing ability is usually investigated via the initial density (or bulk density) $\rho(0)=\rho_{0}$, the final density (or tapped density) $\rho(500)=\rho_{500}$ and the Hausner ratio $H r=\rho(500) / \rho(0)$. These parameters are characterizing the range of densities that can be obtained with the powder as a function of its history. Moreover, the full packing curve provided by the GranuPack allows the definition of dynamical parameters characterizing the kinetics of packing: the number of taps $n_{1 / 2}$ needed to reach one half of the packing amplitude and the initial slope $\alpha$ at the beginning of the process. The initial slope $\alpha$ is computed with a linear fitting $\rho(n)=\rho_{0}+\alpha . n$ on the initial linear part of the packing curve. The higher the initial slope the faster the packing. This dynamical parameter better describes the behavior at the initial stage of the packing for which the grains benefit from the highest mobility to rearrange.

\section{Results and discussion}

The Hausner ratio evolution with temperature is presented in figure 2 for the lactose, cocoa and dried milk powders. The Hausner ratio of lactose powder is not significantly influenced by temperature below $60^{\circ} \mathrm{C}$. Dried milk and cocoa powders both exhibit a decrease of $\mathrm{Hr}$ even at low temperature, particularly for cocoa powder. Indeed, cocoa powder is significantly affected by temperature between $25^{\circ} \mathrm{C}$ and $35^{\circ} \mathrm{C}$, but then exhibits not further decrease of $\mathrm{Hr}$ for higher temperature. The tested cocoa powder contains a large amount of fat $(21.3 \%)$ which may be at the origin of the initial drop of $\mathrm{Hr}$ for low temperature variations. Indeed, previous studies have evidenced that caking induced by molten fat can arise between $30^{\circ} \mathrm{C}$ and $40^{\circ} \mathrm{C}$ for cocoa powders [31]. The plateau in $\mathrm{Hr}$ for tempera- 
ture higher than $35^{\circ} \mathrm{C}$ indicates caking state may already have occurred.

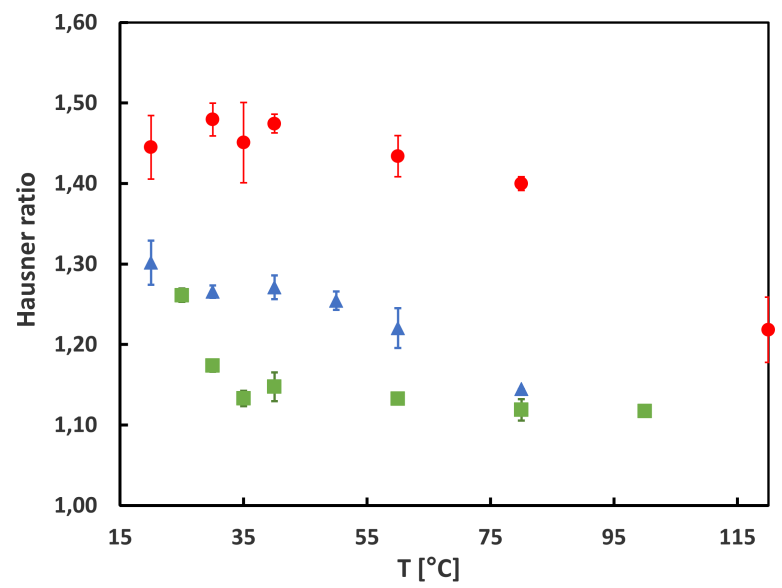

Figure 2. Evolution of the Hausner ratio $(\mathrm{Hr})$ as a function of the temperature for lactose powder (red dots), dried milk (blue triangles) and cocoa (green squares). Error bars are \pm the standard deviation around the mean computed from three repeatability tests.

Classically, a lower Hausner ratio is the signature of a lower cohesiveness. Indeed, a cohesive powder is able to sustain a loose packing at rest due to cohesive interactions opposing the weight of the particles. The opposite behavior is observed here as a higher temperature globally leads to a decrease of the Hausner ratio for the three powders although the grains are expected to become more and more sticky. Ultimately, the sample will reach caking state with the setting of strong solid bridges between the grains preventing packing and thus leading to $H r=1$. No significant changes of the initial density $\rho_{0}$ measured on heated sample have been observed. The apparent decrease of $\mathrm{Hr}$ is thus due to a lower tapped density at higher temperature (see table 1). Changes induced by elevated temperature, probably due to change in particle surface properties, reduce the grain mobility and thus their ability to rearrange during taps and as a consequence the assembly reaches a lower tapped density.

The GranuPack measurement method gives access to the whole packing curve, allowing to investigate changes in the kinematics of packing via the initial slope $\alpha$ measure. The initial slope evolution with temperature is presented in figure 3 . Results show that the temperature increase starts to affect the packing kinematics even for low temperature variation. Indeed, even for a low elevation of temperature from $20^{\circ} \mathrm{C}$ to $30^{\circ} \mathrm{C}$ the decrease of the initial slope, associated to a slow down of the packing, is observed. Temperature thus have a significant effect on the particle cohesive interactions leading to a change of the packing kinematics. Interestingly, despite no change in $\mathrm{Hr}$ for lactose powder below $60^{\circ} \mathrm{C}$, we already observe a slow down of the packing kinematics at low temperature. This indicates that at low temperature the packing ability, i.e. the reachable tapped density, is not influenced by temperature but the dynamics of initial stage of packing is.
Table 1. Final (tapped) density $\rho_{500}$ at $30^{\circ} \mathrm{C}$ and $80^{\circ} \mathrm{C}$.

\begin{tabular}{ccc}
\hline Powder & $\rho_{500}$ at $30^{\circ} \mathrm{C}(\mathrm{g} / \mathrm{ml})$ & $\rho_{500}$ at $80^{\circ} \mathrm{C}(\mathrm{g} / \mathrm{ml})$ \\
\hline Lactose & 0.77 & 0.73 \\
Cocoa & 0.47 & 0.43 \\
Dried Milk & 0.52 & 0.44 \\
\hline
\end{tabular}

Moreover, this demonstrates that the Hausner analysis is not sufficient to give a complete description of the influence of temperature.

The modification of the packing dynamics indicates that significant changes appear at scale of the contact between the grains leading to modifications of inter-grain interactions. For some powders (for example cacao), this could be attributed to a melting at the surface of the grains. However, for other powders, the effect is observed far below the melting temperature. An increase of the temperature can modify the different forces of interaction between the grains through different mechanisms. The Young modulus could decrease with temperature, increasing the surface of contacts and therefore the friction force. In powders containing moisture, a modification of the temperature will modify the water thermodynamic equilibrium inside the powder and induce a set of complex mechanisms. The capillary bridges could evaporate creating possibly solid bridges, the water contained inside the grains could migrate to the surface, the water could experience vaporisation, etc. We have shown in previous studies [25, 26] that the packing dynamics is governed by the mobility of the grains. This mobility is reduced when friction or cohesive forces become stronger. The packing dynamics is therefore slowed down leading to a decrease of the initial slope $\alpha$. Moreover, the increase of the inter-grain forces with temperature reduces the ability of the grains to find configurations optimizing the density. Therefore, the Hausner ratio decreases also with temperature.

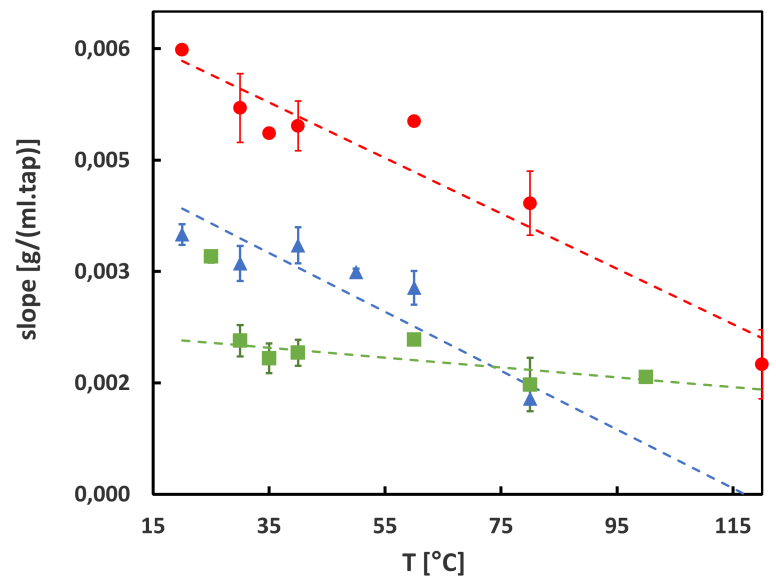

Figure 3. Evolution of the packing initial slope $\alpha$ as a function of the temperature for lactose powder (red dots), dried milk (blue triangles) and cocoa (green squares). Error bars are \pm the standard deviation around the mean computed from three repeatability tests. 


\section{Conclusion}

Temperature has been found to have a significant influence on the packing dynamics of lactose, cocoa and dried milk powders. The Hausner ratio decreases with temperature, indicating an evolution of particle properties even at low temperature. This behavior is more pronounced for cocoa powder, significantly affected at temperature from $25^{\circ} \mathrm{C}$ to $35^{\circ} \mathrm{C}$, which is attributed to the high fat content of this powder leading to caking state at low temperature.

Useful information on the evolution of the packing dynamics have been extracted from the slope of the initial phase of the packing. Indeed, despite a constant $\mathrm{Hr}$ observed up to $60^{\circ} \mathrm{C}$ for lactose powder, the initial slope indicated that the slow down in packing kinematics is already observed at lower temperatures. Hausner ratio analysis thus have to be supplemented with packing dynamics information to get a better picture of the influence of temperature.

Moreover, this exploratory study highlighted the importance of quantifying the influence of the temperature on the behavior of powders, even for small variations of the environmental conditions. Powder characterization including effect of environmental conditions is thus essential to guarantee the quality and consistency of products processed at different processing units all around the globe. The tapped density analysis with packing kinematics evaluation is found to be suitable for this purpose.

Further investigations will be required to understand the changes in the micro properties of the particles as well as particle/particle interactions that appear with temperature elevation. For this purpose, numerical simulations should provide an interesting way to gather knowledge on the physical mechanisms coming into play.

\section{References}

[1] P. G. de Gennes, Rev. Mod. Phys. 71, S374 (1999)

[2] H. M. Jaeger, S. R. Nagel, Science 255, 1524 (1992)

[3] GDR MiDi, Eur. Phys. J. E 14, 341 (2004)

[4] B. Andreotti, Y. Forterre, O. Pouliquen, Granular Media: Between Fluid and Solid (Cambridge University Press, 2013)

[5] D. Geldart, E.C. Abdullah, A. Verlinden, Powder Technol. 190, 70 (2009)

[6] M. Krantz, H. Zhang, J. Zhu, Powder Technol. 194, 239 (2009)

[7] G. Lumay, F. Boschini, K. Traina, S. Bontempi, J.-C. Remy, R. Cloots, N. Vandewall, Powder Technol. 224, 19 (2012)

[8] G. Landi, D. Barletta, M. Poletto, Powder Technol. 207, 437 (2011)
[9] A. Kudrolli, Nat. Mater. 7, 174 (2008)

[10] J. E. Fiscina, G. Lumay, F. Ludewig, N. Vandewalle, Phys. Rev. Lett. 105, 048001 (2010)

[11] E. Emery, J. Oliver, T. Pugsley, J. Sharma, J. Zhou, Powder Technol. 189, 409 (2009)

[12] E. Mersch, G. Lumay, F. Boschini, N. Vandewalle, Phys. Rev. E 81, 041309 (2010)

[13] D. Ruggi, C. Barres, J.-Y. Charmeau, R. Fulchiron, D. Barletta, M. Poletto, Addit. Manuf. 33, 101143 (2020)

[14] M. Van den Eynde, L. Verbelen, P. Van Puyvelde, Influence of temperature on the flowability of polymer powders in laser sintering, in AIP Conference Proceedings 1914 (2017)

[15] G. Lumay, F. Francqui, C. Detrembleur, N. Vandewalle, Adv. Powder. Technol. 31, 4428-4435 (2020)

[16] L. C. Wei, L. E. Ehrlich, M. J. Powell-Palm, C. Montgomery, J. Beuth, J. A. Malen, Addit. Manuf. 21, 201-208 (2018)

[17] J. M. Valverde, A. Castellanos, Europhys. Lett. 75, 985 (2006)

[18] A. Castellanos, J.M. V., A.T. Perez, A. Ramos, P.K. Watson, Phys. Rev. Lett. 82, 1156 (1999)

[19] J. M. Valverde, M. J. Espin, M. A. S. Quintanilla, A. Castellanos, Phys. Rev. E 79, 031306 (2009)

[20] G. Lumay, N. Vandewalle, Phys. Rev. E 78, 061302 (2008)

[21] G. Lumay, S. Dorbolo, N. Vandewalle, Phys. Rev. E 80, 041302 (2009)

[22] G. Lumay, N. Vandewalle, Phys. Rev. E 82, 040301 (2010)

[23] D. Olusanmi, C. Wang, M. Ghadiri, Y. Ding, K.J. Roberts, Powder Technol. 201, 248 (2010)

[24] G. Lumay, N. Vandewalle, Phys. Rev. E 70, 051314 (2004)

[25] G. Lumay, N. Vandewalle, Phys. Rev. Lett. 95, 028002 (2005)

[26] G. Lumay, N. Vandewalle, Phys. Rev. E 74, 021301 (2006)

[27] P. Richard, M. Nicodemi, R. Delannay, P. Ribière, D. Bideau, Nat. Mater. 4, 121 (2005)

[28] G. Lumay, N. Vandewalle, C. Bodson, L. Delattre, O. Gerasimov, Appl. Phys. Lett. 89, 093505 (2006)

[29] Powder flow (European pharmacopoeia, Chapter 2.9.36, 2008)

[30] K. Traina, R. Cloots, S. Bontempi, G. Lumay, N. Vandewalle, F. Boschini, Powder Technol. 235, 842852 (2013)

[31] J. Petit, F. Michaux, C. Jacquot, E. Chavez Montes, J. Dupas, V. Girard, A. Gianfrancesco, J. Scher, C. Gaiani, J. Food Eng. 199, 42-53 (2017) 